\title{
Nalmefene attenuates neural alcohol cue-reactivity in the ventral striatum and subjective alcohol craving in patients with alcohol use disorder
}

\author{
Damian Karl ${ }^{1}$ • J. Malte Bumb ${ }^{1,2} \cdot$ Patrick Bach $^{1,2} \cdot$ Christina Dinter $^{1} \cdot$ Anne Koopmann ${ }^{1,2} \cdot$ Derik Hermann $^{1,2}$. \\ Karl Mann ${ }^{1}$ • Falk Kiefer ${ }^{1,2,3}$. Sabine Vollstädt-Klein ${ }^{1,3}$ (D)
}

Received: 22 October 2020 / Accepted: 29 March 2021 / Published online: 12 April 2021

(C) The Author(s) 2021

\begin{abstract}
Rationale Alcohol use disorder is a common and devastating mental illness for which satisfactory treatments are still lacking. Nalmefene, as an opioid receptor modulator, could pharmacologically support the reduction of drinking by reducing the (anticipated) rewarding effects of alcohol and expanding the range of treatment options. It has been hypothesized that nalmefene acts via an indirect modulation of the mesolimbic reward system. So far, only a few imaging findings on the neuronal response to nalmefene are available.

Objectives We tested the effect of a single dose of $18 \mathrm{mg}$ nalmefene on neuronal cue-reactivity in the ventral and dorsal striatum and subjective craving.

Methods Eighteen non-treatment-seeking participants with alcohol use disorder (67\% male, $M=50.3 \pm 13.9$ years) with a current high-risk drinking level $(M=76.9 \pm 52 \mathrm{~g}$ of pure alcohol per day) were investigated using a cue-reactivity task during functional magnetic resonance imaging (fMRI) in a double-blind, placebo-controlled, cross-over study/design. In addition, selfreported craving was assessed before and after exposure to alcohol cues.

Results An a priori defined region of interest (ROI) analysis of fMRI data from 15 participants revealed that nalmefene reduced alcohol cue-reactivity in the ventral, but not the dorsal striatum. Additionally, the subjective craving was significantly reduced after the cue-reactivity task under nalmefene compared to placebo.

Conclusion In the present study, reduced craving and cue-reactivity to alcohol stimuli in the ventral striatum by nalmefene indicates a potential anti-craving effect of this drug via attenuation of neural alcohol cue-reactivity.
\end{abstract}

Keywords Harm reduction · Reduced drinking · Pharmacotherapy · Controlled drinking · Opioid receptors · Alcohol cue-reactivity $\cdot$ Striatum

Sabine Vollstädt-Klein

s.vollstaedt-klein@zi-mannheim.de

1 Department of Addictive Behaviour and Addiction Medicine, Central Institute of Mental Health, University of Heidelberg, Medical Faculty Mannheim, Mannheim, Germany

2 Feuerlein Center on Translational Addiction Medicine (FCTS), University of Heidelberg, Heidelberg, Germany

3 Mannheim Center for Translational Neurosciences (MCTN), University of Heidelberg, Medical Faculty Mannheim, Mannheim, Germany

\section{Introduction}

Alcohol use disorder (AUD) is one of the most prevalent substance use disorders worldwide (Peacock et al. 2018). However, only $22 \%$ of AUD patients in Europe receive an addiction-specific treatment (Rehm et al. 2015). One possible reason for this low treatment rate could be a lack of willingness for abstinence. Even if abstinence should be the primary goal of addiction treatment, reducing alcohol consumption as a harm reduction may be an alternative treatment option. Against this background, reducing this unwanted treatment gap might be achieved by offering patients to choose between abstinence and reduced drinking as their individual treatment goal (Ambrogne 2002; Batra et al. 2016; Sobell and Sobell 1995). 
Treating alcohol use disorder pharmacologically, disulfiram (approved by U.S. Food and Drug Administration [FDA] but not approved by European Medicines Agency [EMA] anymore), acamprosate (approved by FDA and EMA), as well as naltrexone (approved by FDA and EMA), and nalmefene (approved by EMA) present the currently available and approved options (Soyka and Müller 2017). Naltrexone, which was indicated for relapse prevention, to remain abstinent and to reduce craving in alcohol use disorder (Anton 2008) is a ( $\mu-, \delta$-, and $\mathrm{K}^{-}$) opioid receptor antagonist (Hendershot et al. 2016). By this means, opioid antagonists play a key role in mediating the rewarding effects of alcohol (Gianoulakis 2001), by suppressing the alcohol-induced release of dopamine in the mesolimbic reward system (Spanagel and Weiss 1999; Spanagel and Zieglgansberger 1997), which again might reduce the subjective alcohol craving (Hendershot et al. 2016; O'Malley et al. 1992; Volpicelli et al. 1992). The approval by the FDA was based on these data showing that naltrexone decrease relapse rates to alcohol use, reduced drinking days, and alcohol craving (O'Malley et al. 1992; Volpicelli et al. 1992) probably by reducing the positive reinforcing effects of alcohol and/or the anticipation of such effects (Heilig et al. 2010). This is also reflected in a decreased fMRI cue-reactivity by naltrexone in the ventral striatum of non-treatment-seeking alcoholics (Myrick et al. 2008). The ventral striatum has been associated to motivational reward processes, which is manifested in a greater activation by alcohol respectively reward-associated stimuli, while the dorsal striatum has been linked to stereotyped and automated behavior (Braus et al. 2001; Everitt and Robbins 2005; Everitt and Robbins 2016; Schacht et al. 2013). Also, imaging studies indicate that there is a shift from ventral to dorsal cue processing (Vollstädt-Klein et al. 2010) and overreliance on habitual learning with increased activation in dorsal striatum in the presence of alcohol dependence (Sjoerds et al. 2013) in the course of addiction development. Against this background, cue-induced brain activation measured with fMRI in the striatum and the ACC was associated with an increased amount of drinking at follow-up (Courtney et al. 2016; Grüsser et al. 2004). In addition to these neurobiological findings, a metaanalysis of Jonas et al. (2014) showed that the numbers needed to treat for benefit (NNTs) for naltrexone $(50 \mathrm{mg} /$ day) were 20 to prevent return to any drinking and 12 to prevent return to heavy drinking. Considering this, recently, nalmefene has been approved by the EMA specifically for the reduction of alcohol consumption in adult patients suffering from AUD and a high drinking risk level (> $60 \mathrm{~g}$ pure alcohol on a single drinking day for men and $>40 \mathrm{~g}$ for women), without physical withdrawal symptoms and not requiring immediate detoxification (Online document. Online document. European Medicines Agency [EMA] 2013). In 2005, about 11 million people in Europe aged between 18 and 64 years suffered from alcohol dependence, and in $2009,15 \%$ of men and $8 \%$ of woman consumed alcohol at a high or very high risk level in Europe (Rehm et al. 2012) according to which nalmefene would address a sizeable group.

Nalmefene acts as an opioid system modulator with antagonistic activity at the $\mu$-and $\delta$-receptors, as well as naltrexone, and partial agonistic activity at the $\mathrm{K}$-receptor (Bart et al. 2005), distinguishing the drug from other drugs that act within the opioid system, such as naltrexone which is a full kappa receptor antagonist (Swift 2013; Vollstädt-Klein et al. 2019).

Besides this different pharmacological principle, the treatment with nalmefene also differs from previous treatment strategies. While therapy with naltrexone takes place continuously, nalmefene should be taken as needed before high-risk drinking situations (Online document. Online document. European Medicines Agency [EMA] 2013). On this occasion, nalmefene should reduce the reinforcing effect of alcohol due to its influence on the mesolimbic reward system, alleviating the reduction of alcohol consumption.

So far, previous investigations showed that nalmefene as on-demand medication is superior to placebo in reducing the number of heavy drinking days (Gual et al. 2013; Mann et al. 2013; Mann et al. 2016; van den Brink et al. 2013). More recent investigations have also shown that nalmefene given as needed reduces the number of heavy drinking days after 12 weeks compared to placebo (Miyata et al. 2019) and treatment as usual (Castera et al. 2018). One further study Quelch et al. (2017) demonstrated that nalmefene reduces in the presence of the alcohol infusion the neuronal response regarding the anticipation of reward in striatal areas if compared to placebo.

Nevertheless, hitherto, the neurobiological mechanism of action of nalmefene is not well understood and needs further investigation. Therefore, we investigated the effect of single-dose nalmefene on neural alcohol-cue-reactivity during fMRI and subjective alcohol craving. We hypothesized that a single dose of nalmefene is superior over placebo in decreasing neural reactivity in the ventral and dorsal striatum, following the presentation of alcohol associated visual stimuli. This would suggest that nalmefene can dampen the hedonistic effects of alcohol or counteract the anticipation of this effect. Due to the shift from ventral to dorsal striatal cue processing (Vollstädt-Klein et al. 2010) and preliminary fMRI findings that show that opiate antagonists like naltrexone and nalmefene are able to reduce brain activity in mesolimbic pathway after cue exposure (Bach et al. 2020; Myrick et al. 2008; Quelch et al. 2017), we concentrate in particular on the ventral and dorsal striatum.

\section{Methods and materials}

The effect of a single-dose nalmefene $(18 \mathrm{mg}$ ) on cuereactivity was examined prospectively, using a double-blind, placebo-controlled study design (cross-over design) and functional magnetic resonance imaging (fMRI) (registration at 
clinicaltrials.gov; NCT02372318). Heavy-drinking (alcohol consumption $>60 \mathrm{~g}$ for men and $>40 \mathrm{~g}$ for women; at least 5 days/week), non-treatment-seeking participants with alcohol use disorder ( $\geq$ five DSM-5 AUD criteria) were selected as the target population. Recruitment was conducted through local notices, several press releases, advertisements, and bulletin in social media. All participants provided written informed consent prior to study participation.

\section{Study design}

The study consisted of a telephone screening, a baseline screening, and two investigational days including fMRI examination of cue-reactivity (see Fig. 1). Participants had to meet all following inclusion criteria in order to take part in the study: (1) participants had to be aged between 18 and 70 years; (2) they had to meet at least five diagnostic criteria for an alcohol use disorder according to the Diagnostic Statistical Manual of Mental Disorders (DSM-5); (3) the average amount of consumed pure alcohol should be at least $\geq 60 \mathrm{~g}$ for men and $\geq 40 \mathrm{~g}$ for women per day (at least 5 days/week); (4) they should have a sufficient visual acuity (binocular [corrected] $\geq$ 0.8 ). Participants were excluded if they met one or more of the following exclusion criteria: (1) previous inpatient detoxification treatment; (2) current withdrawal symptoms (CIWA-Ar > 4; Sullivan et al. 1989), previous severe withdrawal or any withdrawal complications; (3) other Axis I psychiatric diagnoses than alcohol or tobacco use disorder in the last 12 months screened with Structured Clinical Interview (SKIDI) for DSM-4 (Wittchen et al. 1997) due to the unavailability of a SKID for DSM-5 at the time of examination; (4) neurological disorders respectively history of brain injury; (5) at the time of the examination psychotropic medication within the last 14 days; (6) an intoxication (breath alcohol concentration $>0.3 \%$ ); (7) positive drug screening (opioids, cannabinoids, benzodiazepines, barbiturates, cocaine, amphetamines); (8) positive pregnancy test or (9) contraindications to the prescription of nalmefene (e.g., known intolerance, current use of opioid analgesics or opioid-containing antidiarrheal, positive opioid in urine, opiate withdrawal syndrome, or severe liver dysfunction); and (10) exclusion criteria for MRI (e.g., metal implants and claustrophobia).

On screening day, participants were informed about study procedures and possible risks such as medication side effects or headaches after fMRI. Basic sociodemographic information was documented, and history of somatic illnesses and neurological and mental disorders as well as current medication was recorded. Health status was assessed by medical examination. Absence of current illicit drug abuse was verified via urine screening; for women, a pregnancy screen was conducted additionally. An alcohol breath test was performed to confirm abstinence (breath alcohol concentration $>0.3$ ). After medical check-up, participants underwent the Structured Clinical Interview for DSM-4 to verify the absence of Axis-I disorder (SKID-I; Wittchen et al. 1997). This interview was chosen as there was no SKID interview available for DSM-5 at the time of the investigation. Alcohol consumption during the last 3 months was recorded using the Form 90 interview (Scheurich et al. 2005), as well as nicotine status and consumption during the last 3 months. Psychometric assessment comprised the Fagerstrøm Test of Nicotine Dependence (FTND; Heatherton et al. 1991), the Alcohol Dependence Scale (ADS; Skinner and Allen 1982), the Inventory of Drinking Situations (IDS; Annis et al. 1987), the Beck Depression Inventory (BDI; Beck et al. 1961), and the Alcohol Use Disorders Identification Test (AUDIT; Reinert and Allen 2002), recorded via an electronic platform (Social Science Survey, www.soscisurvey.de).

At the investigational day, participants underwent a medical check-up for occurrence of somatic illnesses representing a contraindication for assessment or nalmefene intake. An alcohol breath test was performed to ensure abstinence. Current withdrawal symptoms were captured with the Clinical Institute Withdrawal Assessment for alcohol scale (CIWAAr; Sullivan et al. 1989). After assuring the absence of any exclusion criteria valid at MRI investigation, study medication

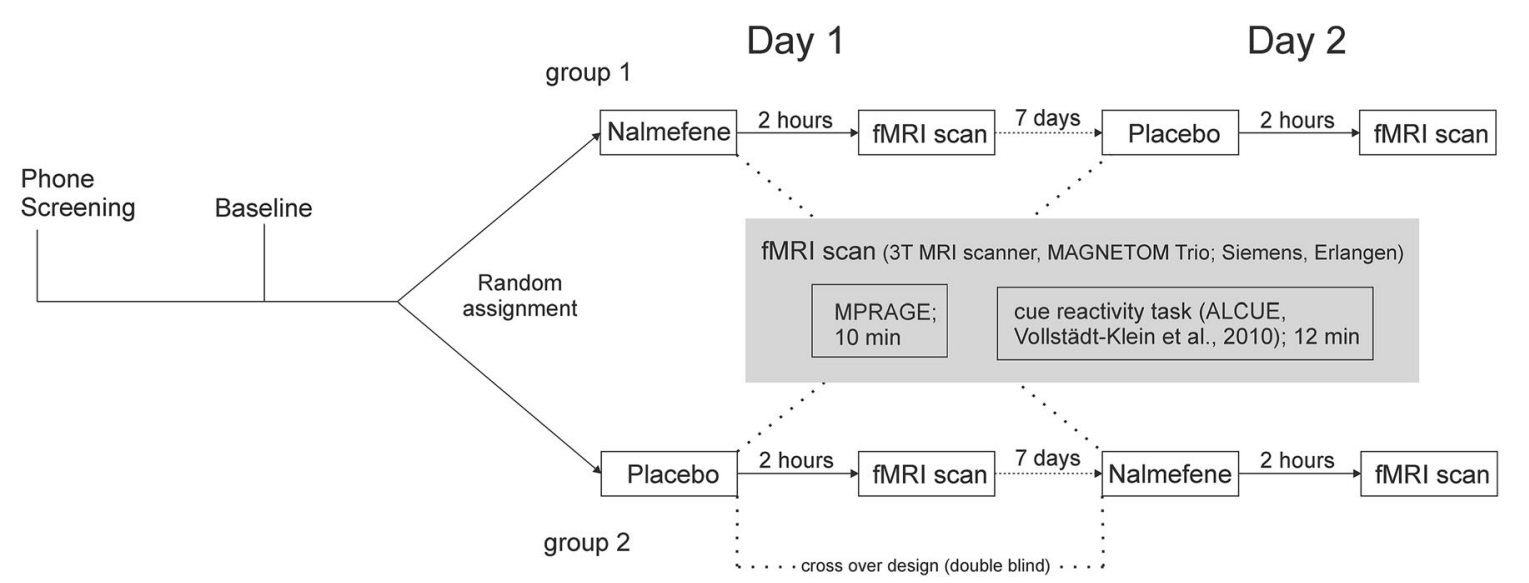

Fig. 1 Study procedures 
was handed out to the participant, and intake was supervised. Alcohol consumption since baseline measurement or investigational day one respectively was recorded using the Form 90 interview (Scheurich et al. 2005). A second investigational day was scheduled at a 1-week interval and differed only in the double-blind, randomized, oral administration of nalmefene $(18 \mathrm{mg})$ or placebo $2 \mathrm{~h}$ before the fMRI measurement. These timeframes were determined on the basis of the pharmacokinetic parameters for nalmefene, namely a time to peak concentration of $0.8 \mathrm{~h}$ (median) and opioid receptor occupancy up to $74 \mathrm{~h}$ after oral administration of $(20 \mathrm{mg})$ nalmefene (Ingman et al. 2005). Participants were then discharged for a recreational period until a 2 -h period after medication intake was fulfilled to ensure substance invasion. Before fMRI measurement, participants' severity of craving was captured via paper-pencil assessment applying the Alcohol Urge Questionnaire (AUQ, 8 items on a seven-point rating scale; Bohn et al. 1995) and the Alcohol Craving Questionnaire (ACQ, 30 items on a seven-point rating scale; German version, Raabe et al. 2005). Subsequently, participants underwent fMRI measurement comprising (1) our alcohol cue-reactivity task (Vollstädt-Klein et al. 2010) and (2) an emotional faces task, which has been analyzed elsewhere (Vollstädt-Klein et al. 2019). Directly afterwards the fMRI scan, the questionnaires (AUQ, ACQ) were applied one more time. After a quick medical check-up by a physician, participants were dismissed. Test for successful blinding was conducted by asking the participants about their estimation for the time point of the medication administration $(56 \%$ did not make any estimate, $39 \%$ correctly identified it, and $6 \%$ made an incorrect estimate).

\section{Alcohol cue-reactivity task}

For the assessment of neural response to alcohol-related stimuli, a cue-reactivity task (ALCUE, Vollstädt-Klein et al. 2010) was used. In this task, 60 alcohol-related and 45 neutral stimuli were presented using a blocked design with five stimuli each block. Each image was presented for $4 \mathrm{~s}$, so each block took $20 \mathrm{~s}$. Alcohol-related pictures were taken from a validated picture series (Vollstädt-Klein et al. 2010), and neutral control cues were taken from the International Affective Picture System. Following each block, the participants were asked for the current intensity of their alcohol craving on a visual analogue scale (VAS) ranging from 0 (no craving) to 100 (extremely extensive craving). Task duration was $12 \mathrm{~min}$.

\section{Functional MRI acquisition}

Functional and anatomical brain images were acquired using a 3T whole-body tomograph (MAGNETOM Trio, Siemens Medical Systems, Erlangen, Germany). Task-related blood oxygen level-dependent (BOLD) response was measured using T2*-weighted echo planar imaging (EPI) sequences $\left(\mathrm{TR}=2.41 \mathrm{~s}, \mathrm{TE}=25 \mathrm{~ms}\right.$, flip angle $=80^{\circ}, 42$ slices, slice thickness $2 \mathrm{~mm}, 1 \mathrm{~mm}$ gap, voxel dimensions $3 \times 3 \times 3 \mathrm{~mm}^{3}$, FOV $192 \times 192 \mathrm{~mm}^{2}, 64 \times 64$ in-plane resolution). The T2*weighted EPI sequences were acquired in a transversal orientation $30^{\circ}$ clockwise to AC-PC-line covering the whole brain. This short $\mathrm{TE}$ and the $30^{\circ}$ flip to AC-PC orientation were chosen to minimize susceptibility artifacts. The number of images measured at the ALCUE Task for each participant was 305 .

In addition, high-resolution anatomical scans using T1weighted 3-D magnetization-prepared rapid acquisition gradient-echo (MPRAGE) sequences consisting of 192 sagittal slices (slice thickness $1 \mathrm{~mm}, 1 \times 1 \times 1 \mathrm{~mm}$ voxel size, FOV $256 \times 256 \mathrm{~mm}^{2}, \mathrm{TR}=2300 \mathrm{~ms}, \mathrm{TE}=3.03 \mathrm{~ms}, \mathrm{TI}=900 \mathrm{~ms}$, flip angle $=9^{\circ}$ ) were acquired for each participant.

\section{fMRI pre-processing}

Pre-processing and statistical analyses of brain imaging data were performed using SPM8 (Wellcome Department of Cognitive Neurology, London, UK). The first five scans were excluded from the analyses to avoid artifacts due to magnetic saturation effects. The remaining scans were realigned spatially to correct for head motion over the course of the session and then normalized to an MNI (Montreal Neurological Institute, Quebec, Canada) EPI template. Subsequent smoothing was performed using an isotropic Gaussian kernel for group analysis $(8 \mathrm{~mm}$ full width at half maximum [FWHM]).

\section{Statistical analysis}

Statistical analyses of the pre-processed fMRI data on the first (individual) level were performed by modeling the different conditions (alcohol-associated versus neutral control stimuli; boxcar functions convoluted with the hemodynamic response function) as explanatory variables within the context of the general linear model (GLM) on a voxel-by-voxel basis with SPM8. Realignment parameters were included as regressors of no interest. The following contrast images were calculated for each participant and each drug condition: (1) favorite drink

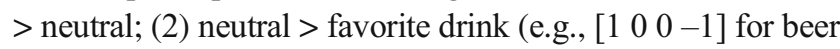
drinkers and $\left[\begin{array}{llll}1 & 1 & 0 & -2\end{array}\right]$ for beer and wine drinkers).

Individual contrast images described above of the participants were included in a second level analysis (full factorial model) to identify the main effects as well as differences between the verum and the placebo condition. The sequence of drug administration (placebo first/nalmefene first) was considered as a covariate of no interest in the analysis. We conducted a region of interest (ROI) analysis because of the strong a priori hypotheses previously defined in the study protocol (clinicaltrials.gov, NCT02372318) of cue-induced activation of the ventral (VS) and the dorsal striatum (DS) especially due 
to previous findings of reduce brain activity in mesolimbic regions after intake of opiate antagonists and cue exposure (Myrick et al. 2008; Quelch et al. 2017). The ROI mask for the ventral striatum was created by placing two $10 \mathrm{~mm}$ spheres bilaterally on the MNI coordinates $[+-12,8,-8]$. These coordinates were determined using a term-based metaanalyses search at the platform www.neurosynth.org. For the dorsal striatum, a self-created and already established mask of Vollstädt-Klein et al. (2010) was used (see Fig. 2). For the ROI analyses, FWEcorrected $p$-values are reported at cluster level.

In addition to the ROI analysis, we conducted a whole brain analysis for exploratory analysis. To control for multiple statistical testing, the probability of a family wise error (FWE) was set to .05. For this purpose, we used the AlphaSim (3dClustSim) method. A voxel wise threshold of $p<.02$ was combined with a cluster extent threshold of 795 (placebo, contrast favorite drink $>$ neutral), 882 (nalmefene, contrast favorite drink > neutral), and 841 voxels (placebo > nalmefene, contrast favorite drink $>$ neutral), determined by the AlphaSim using 25000 Monte Carlo simulations for the whole brain analysis of the ALCUE task. Estimation of smoothness based on the residual images was conducted using SPM by taking the maximum of the 3 estimated parameters in $\mathrm{x}, \mathrm{y}$, and $\mathrm{z}$ directions.

The self-reported craving was analyzed in SPSS (Statistical Package of the Social Sciences, version 24; SPSS; IBM Corporation, Armonk, NY, USA) using paired $t$-tests.

\section{Results}

Twenty-three participants were randomized to one of the two groups; one group was administered nalmefene at the first time point, and in the other one, nalmefene was given at the second time of measurement. Of the 23 randomized participants, ten participants (44\%) reported adverse side effects (five participants of group 1 and five participants of group 2). A mean of $5.8(S D=2.7)$ symptoms occurred and lasted a mean time of $37.5 \mathrm{~h}(S D=22.3 \mathrm{~h})$. For a detailed overview of the adverse side effects that occurred, see Supplement Table 1, Reported side effects.
Fig. 2 Masks for region of interest (ROI) analysis, ventral striatum (red) two $10 \mathrm{~mm}$ spheres bilaterally on the MNI coordinates $[+-12,8,-8]$; the dorsal striatum (blue) self-created and already established mask of Vollstädt-Klein et al. (2010)
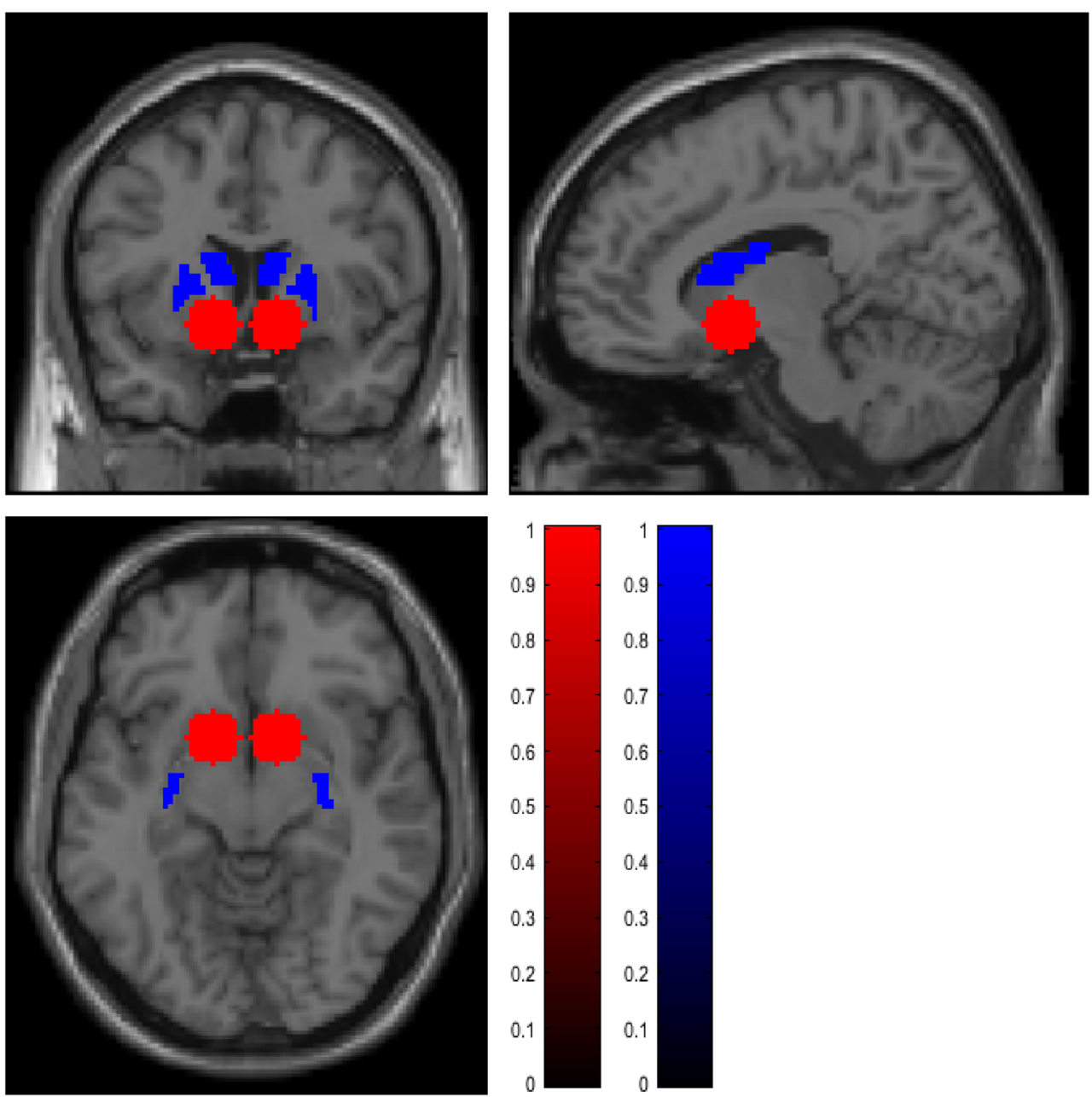
Five individuals had to be excluded from the analyses because of either withdrawal of informed consent (in most cases due to adverse side effects of nalmefene, e.g., insomnia, vertigo, and nausea, $n=4$ from group 1) or unsuitability for fMRI scanning ( $n=1$, metal implant). Therefore, the final sample consisted of 18 participants who finished the whole experimental procedure. Because of technical problems $(n=1)$, or too much head movement $(n=2)$, only 15 participants were included in the final fMRI-data analysis. However, the sample of the 18 participants was used to evaluate the behavioral data. Of these 18 participants, 12 were male $(67 \%)$, and 10 of them were smokers $(59 \%)$. The mean age was 50.3 years $(S D=$ 13.9 years $)$, and a mean of $6.4(S D=1.4)$ DSM-5 criteria for alcohol dependence was met. On average, participants consumed $6.4(S D=4.3)$ standardized alcoholic beverages (12 g each per drink) per day, for a mean amount of $76.9(S D=52)$ grams per day. In the 90 days before the baseline examination, participants had an average of $54(S D=31.6)$ heavy drinking days. On the ADS, participants scored an average of $8.7(S D=4.4)$. For the extent of harmful use and alcohol dependence (AUDIT), the mean score was $17.2(S D=5.8)$. For detailed demographics information of these 18 respectively and 15 participants, see Table 1 .

\section{fMRI results}

Overall, data of 15 heavy drinkers was compared between the conditions nalmefene and placebo. There was a significant lower neural cue-reactivity (favorite drinks $>$ neutral) in the ventral striatum (a priori ROI) in the nalmefene condition compared to the placebo condition $[(\mathrm{x}, \mathrm{y}, \mathrm{z})=(-4,4,-8), t$ $=6.20, p_{F W E \text { corrected }}=.007 ;(\mathrm{x}, \mathrm{y}, \mathrm{z})=(4,4,-10), t=4.50, p$ $F W E$ corrected $=.032]$; see Fig. 3 . Such a difference could not be observed for the dorsal striatum. For the ventral striatum, the actual data demonstrated an effect size of $d=0.74$ resulting in a power of $86 \%$. Aside from that, there was no main effect for the contrast alcohol (fav) $>$ neutral in the ROI analyses for placebo or nalmefene condition.

However, the explorative whole brain analysis (i.e., nonROI-based approach) revealed a broadly activated network (for the contrast favorite drink > neutral) including striatal areas, limbic regions (hippocampus and anterior cingulate), and inferior and middle frontal gyrus under placebo, but not under the nalmefene condition (see for a detailed overview Supplement material Fig. 3 and Supplement Tables 2 and 3).

In addition, the exploratory whole brain analysis for the direct comparison placebo vs. nalmefene showed an interaction effect, driven by a decreased activation under nalmefene (i.e., placebo $>$ nalmefene and favorite drink $>$ neutral) in
Table 1 Effects of single-dose nalmefene on cue-reactivity and craving in alcohol use disorder

\begin{tabular}{|c|c|c|c|c|c|c|}
\hline & \multicolumn{3}{|c|}{$\begin{array}{l}\text { Investigation completed } \\
(n=18)\end{array}$} & \multicolumn{3}{|c|}{$\begin{array}{l}\text { Cue-reactivity task } \\
\text { completed }(n=15)\end{array}$} \\
\hline & $M$ & $S D$ & $n(\%)$ & $M$ & $S D$ & $n(\%)$ \\
\hline Age & 50.3 & 13.9 & & 52.3 & 14.1 & \\
\hline \multicolumn{7}{|l|}{ Sex } \\
\hline Male & & & $12(67 \%)$ & & & $10(67 \%)$ \\
\hline Female & & & $6(33 \%)$ & & & $5(33 \%)$ \\
\hline Smoker & & & $10(56 \%)$ & & & $7(47 \%)$ \\
\hline Drinks per day $^{\mathrm{a}}$ & 6.4 & 4.3 & & 6.8 & 4.6 & \\
\hline Amount of pure alcohol per day (grams) ${ }^{b}$ & 76.9 & 51.8 & & 81.1 & 55.3 & \\
\hline Abstinent days ${ }^{\mathrm{c}}$ & 21.2 & 22.9 & & 20.4 & 22.2 & \\
\hline Heavy drinking days $(>48 \mathrm{~g} \text {, female } />60 \mathrm{~g}, \text { male })^{\mathrm{c}}$ & 54.0 & 31.6 & & 53.8 & 31.1 & \\
\hline Number of fulfilled DSM- 5 criteria for AUD & 6.4 & 1.4 & & 6.4 & 1.4 & \\
\hline ADS & 8.7 & 4.4 & & 8.5 & 4.7 & \\
\hline AUDIT & 17.2 & 5.8 & & 17.4 & 6.1 & \\
\hline BDI & 6.2 & 6.1 & & 6.1 & 6.2 & \\
\hline FTND $^{\mathrm{d}}$ & 3.4 & 3.0 & & 3.3 & 2.7 & \\
\hline
\end{tabular}

Notes. $M$ mean, $S D$ standard deviation, ADS Alcohol Dependence Scale, AUDIT Alcohol Use Disorders Identification Test, $B D I$ Beck Depressions Inventory, FTND Fagerström Test for Nicotine Dependence

${ }^{\mathrm{a}} 12 \mathrm{~g}$ per drink

${ }^{\mathrm{b}}$ Conversion formula: amount in $\mathrm{ml} *($ Vol.- $\% / 100) * 0.8=$ gram pure alcohol

${ }^{\mathrm{c}}$ Refers to the 90 days before baseline

${ }^{\mathrm{d}}$ Refers to the subgroup of smokers ( $n=10$ resp. 7) 


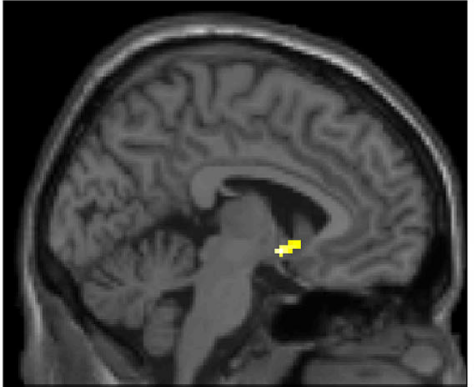

$x=-4$

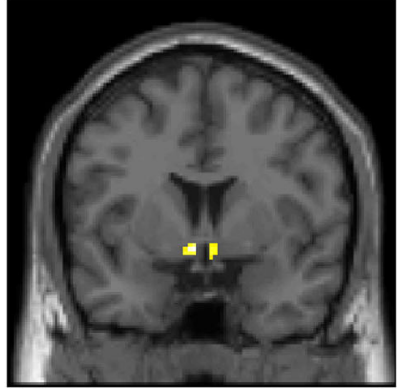

$y=4$

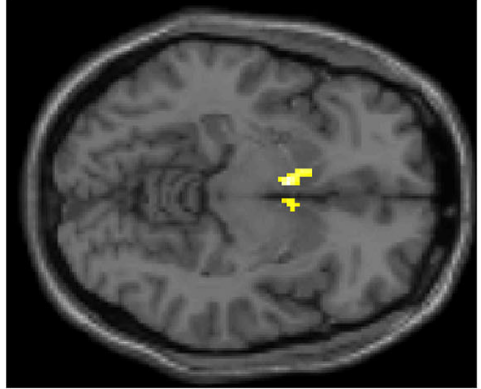

$z=-8$

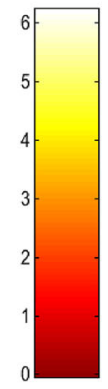

Fig. 3 Results of ROI analysis for ventral striatum; $p<.001$ (uncorr), 10 voxel, contrast: placebo $>$ nalmefene, alcohol (favorite) $>$ neutral middle frontal gyrus, anterior cingulate, postcentral gyrus, superior frontal gyrus, and middle temporal gyrus (see in detail Supplement Table 4). There was no significant increase in brain activation in any brain region by nalmefene.

\section{Subjective craving data (behavioral data)}

No significant difference in pre-scanning AUQ or ACQ craving between the nalmefene and placebo condition was present. However, participants reported significantly lower cueinduced craving (measured by AUQ) directly after the ALCUE fMRI task in the nalmefene compared to the placebo condition $(t=1.79, p=0.046$; mean \pm SD nalmefene: $12.82 \pm$ 5.02 [range: $8-38$ ], placebo: $15.35 \pm 5.52$ (range: $8-24$ ); $d=$ $0.479, r=0.41$; see Fig. 4). For the ACQ craving, no such difference could be observed after the fMRI session $(t=0.66$, $p=0.26$; mean \pm SD nalmefene: $42.47 \pm 17.08$ and placebo: $45.12 \pm 19.81)$. An overview of the raw values can be found in Supplement Table 5, Means and standard deviations of the ACQ and AUQ.

Regarding the alcohol craving measured during the cuereactivity task using a VAS ranging from 0 (no craving) to 100 (extremely extensive craving), we found no significant differences between the drug conditions neither for the craving after favorite alcohol cues $(t=0.65, p=0.53$; mean $\pm \mathrm{SD}$

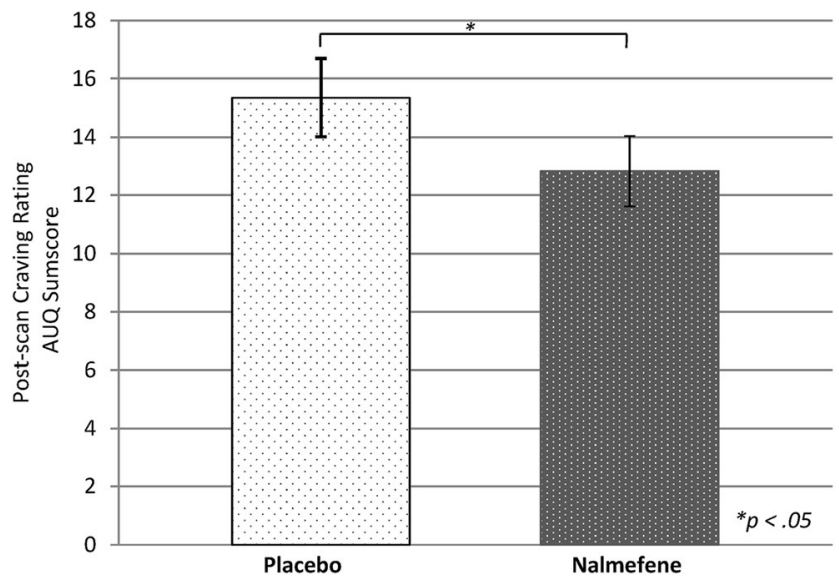

Fig. 4 Comparison sum score of AUQ after fMRI cue-reactivity task between nalmefene and placebo placebo: $26.46 \pm 29.51$ and nalmefene: $22.45 \pm 28.45)$ nor after neutral cues $(t=-1.01, p=0.33$; mean \pm SD placebo: $08.83 \pm 14.98$ and nalmefene: $10.96 \pm 12.98$ ). Also the difference score (favorite alcohol cues - neutral cues) did not differ significantly under either the placebo (mean \pm SD: $17.63 \pm$ 21.17 ) or the nalmefene (mean \pm SD: $11.49 \pm 20.87$ ) condition, $t=1.04, p=0.32$.

Linear correlations between questionnaires and VS and DS activations and linear correlations between difference scores (verum versus placebo condition) were examined. There were no significant correlations between (changes in) neural cuereactivity responses and (changes in) craving.

\section{Discussion}

To the best of our knowledge, we are the first to show that a single-dose nalmefene reduces the neuronal response to alcohol associated stimuli in the ventral striatum. Nalmefene seems to influence neuronal brain responses, responsible for reward-associated behavior (Schultz et al. 1997; Wrase et al. 2007) and may affect substance-related behavior consequently. Such an effect was not revealed for the dorsal striatum. In addition, we could show a reduced subjectively reported craving measured by AUQ after a cue-reactivity task under nalmefene compared to placebo.

This finding expands the results of a first fMRI study by Quelch et al. (2017) who demonstrated similar to the present investigation decreased brain activation in the mesolimbic system under the influence of nalmefene during reward anticipation. In contrast to the present study, Quelch et al. (2017) used a monetary incentive delay task during an intravenous alcohol challenge. We on the other hand administered nalmefene $2 \mathrm{~h}$ before the presentation of alcohol-related stimuli respectively alcohol consumption according to a preventive approach as recommended in the European public assessment report (EPAR) for Selincro® (Online document. Online document. European Medicines Agency [EMA] 2013). This naturalistic approach reflects the use of nalmefene as medication for the reduction of alcohol consumption during confrontation with alcohol-associated stimuli. 
Furthermore, one possible explanatory approach for the reduced brain activation in the ventral striatum but not in the dorsal striatum after the administration of nalmefene could be an earlier stage of alcohol use disorder in the examined sample. The participants scored an average of $8.7(S D=4.4)$ on the ADS (Skinner and Allen 1982). This mean score corresponds to the cut off proposed by Ross et al. (1990) indicating alcohol dependence. For the extent of harmful use and alcohol dependence (AUDIT; Reinert and Allen 2002), the mean score was $17.2(S D=5.8)$, indicating a high level of alcohol-related problems (cut-off $>16$, Babor et al. 2001). It has been hypothesized that a "ventro-dorsal shift" characterizes the switch from "choice to habit" on a neural basis mirroring the progression of the alcohol dependence (Everitt and Robbins 2005; Everitt and Robbins 2016; Vollstädt-Klein et al. 2010). In the present study, all participants met DSM-5 criteria for AUD. However, none of the participants received any previous (semi-) inpatient detoxification treatment. In addition, in the present sample, the mean ADS score and an average of $81 \mathrm{~g}(S D=55.3)$ of consumed pure alcohol per day may indicate that the investigated individuals are "between" light and heavy drinkers and therefore are not yet fully habitual- but rather reward-motivated. This might suggest that especially individuals in early stages of alcohol use disorder could benefit from an add-on therapy with nalmefene. Thus, the results found here suggest that nalmefene could be an agent supporting regaining control over substance use, especially at an early stage of substance use disorder. This approach to regaining control in early stages of substance use disorders (SUD) needs further investigation because it has not yet been sufficiently examined (Heinz et al. 2020).

In a further analysis of Vollstädt-Klein et al. (2019), we investigated the effect of nalmefene on neural activation using fMRI during the presentation of emotional faces pictures in this sample. Results of this suggest that nalmefene is able to influence neuronal processes responsible for social skills and empathy. According to this, nalmefene could have additional useful effects, in addition to its anti-craving effect, especially in the social context, which in turn could support the reduction in amount of drinking (Vollstädt-Klein et al. 2019).

Intriguingly and complementing the above described findings, previous studies have shown that increased activation of the ventral striatum by alcohol-associated cues was associated with an increased risk of relapse in alcohol-dependent individuals. This also suggests that cue-induced activation of ventral striatum might increase the risk of relapse (Braus et al. 2001; Grüsser et al. 2004; Heinz et al. 2009). Against this backdrop, it has to be emphasized that a reduction of the cue-induced brain activation in the ventral striatum through nalmefene might reduce the risk of relapsing into severe drinking patterns.

For the structurally similar opiate antagonist naltrexone, a reduced neuronal activity on alcohol stimuli in the ventral striatum could be demonstrated by fMRI investigation likewise
(Myrick et al. 2008) which is also expressed on the behavioral level. Treatment with naltrexone reduces both subjectively craving and the amount of consumed alcohol compared to placebo treatment (Hendershot et al. 2016; Rösner et al. 2010).

Moreover, in the present study, a significantly lower selfreported craving, as measured with the AUQ, after the fMRI scan was revealed in the nalmefene condition, compared to placebo. This finding could be based on the fact that the AUQ is useful to capture substance cravings before and after a cue-reactivity task (MacKillop 2006), and that this questionnaire is a quick self-report instrument $(<1 \mathrm{~min})$ to assess craving with high internal consistency and high test-retest reliability (Drobes and Thomas 1999). In addition, the AUQ was shown to correlate significantly positively with alcohol dependence severity and with scores on the Obsessive Compulsive Drinking Scale indicates construct validity (Bohn et al. 1995). Also, de Laat et al. (2019) reported significantly reduced alcohol craving as measured by AUQ after naltrexone administration which was also associated with kappa opioid receptor (KOR) availability. This is also in line with our finding, due to a partial agonistic activity at the kappa opioid receptor of nalmefene. Taking together, the reported finding might indicate that nalmefene is able to reduce craving and thus the hedonic effect of alcohol. The particular strength of the within-subjects study design is that each participant serves as their own control and thus reduces the error variance (Bakeman and Robinson 2005).

On the other hand, not all craving measurements showed significant reduction by nalmefene. An explanation for low and not significant different craving measured by ACQ and the fMRI task between the two conditions may also be an effect of social desirability. Even if direct questioning about current substance demand is a common approach, this can be distorted by social desirability (Wiers and Heinz 2015). Additionally, many participants reported that the investigation took place at a time (in most cases, during the day) that was outside of their usual time to drink (in the evening). This aspect in combination with the fact that the participants were in an examination situation could have additionally influenced the self-reported craving. In addition, the ACQ measures the aspect of loss of control relatively insufficiently, so that a multidimensional craving assessment is recommended by combining it with other measures such as the OCDS (Raabe et al. 2005). Future studies could use additional (indirect) measurements of physiological outcomes, such as skin conductance or heart rate, to support the measurement of craving (Drobes and Thomas 1999). Moreover, this could be useful as there is evidence that not all individuals can consciously perceive their substance craving (Tiffany and Conklin 2000), which could be problematic when using self-report measures.

Unexpectedly, there was no main effect for the type of stimulus in the ROI analysis, i.e., there was no increased striatal activation during favorite alcoholic stimuli under the nalmefene or the placebo condition. However, the explorative whole brain analysis with a more liberal threshold indicated 
that alcohol stimuli induced increased activation compared to neutral stimuli in the ventral and dorsal striatum. Here, also other relevant brain regions implicated in cue and reward processing as middle frontal gyri and anterior cingulate cortex (Lukas et al. 2013; Schacht et al. 2013) were shown to be increased in the whole brain analysis under placebo and reduced by nalmefene.

In addition to the lack of observable main effects in the ROI analysis for the type of stimulus, the small sample size should be mentioned as a further weakness of this study. On this account, small effects may have been lost. In addition, the type of stimuli may not be suitable for every participant to provoke cue-reactivity (Mucha et al. 2008). Although we analyzed the contrast "favorite drink > neutral," the picture stimulus set might not have been optimal for each participant.

Another limitation in this specific sample is an unequal distribution of participants between the two groups (see Fig. 1 and group $1: n=5$, group $2: n=10$ ). This is partly explained by dropout due to adverse side effects (e.g., insomnia, vertigo, and nausea; for detailed description, see Supplement Table 1, Reported side effects). In the group of participants who received the nalmefene at the first time point (group 1), four participants dropped out because of adverse side effects, while in group 2 (placebo T1, nalmefene T2), no dropout after the first time point occurred (see Supplementary Figure 1 CONSORT flow diagram). Therefore, drop-out might be affected by medication, which could limit the results as an influence of the sequence of drug administration.

Nevertheless, further research is needed to assess long-term effects of nalmefene on neuronal response since even nonmedical interventions like cue-exposure therapy led to reductions in cue-reactivity (Mellentin et al. 2017).

Taken together, nalmefene attenuated alcohol cuereactivity in the ventral striatum of non-treatmentseeking AUD patients compared to placebo and reduced subjective alcohol craving in our study, supporting the anti-craving effect of this drug. So our findings support the assumption that nalmefene might reduce the amount of drinking via attenuation of neural alcohol cuereactivity.

As previous studies suggested that especially individuals with a high cue-reactivity in the ventral striatum (Mann et al. 2014; Schacht et al. 2017) might particularly benefit from treatment with opioid-antagonists, future studies could establish predictors for successful treatment with nalmefene.

Supplementary Information The online version contains supplementary material available at https://doi.org/10.1007/s00213-021-05842-7.

Acknowledgements We would like to thank Amelie Otto and Michael Riess for their assistance in data collection, Sabine Hoffmann for her assistance in data management, and Alycia Lee for her assistance in data analysis.
Author contribution SVK, KM, and FK were responsible for the study design. DK and CD contributed to the acquisition of $\mathrm{PMRI}$ and psychometric data. DK and SVK performed the data analysis. DK, SVK, PB, and JMB interpreted the data. DK and SVK drafted the manuscript. AK and $\mathrm{DH}$ contributed to the recruitment of patients and conducted physical examinations. All authors revised the manuscript critically for important intellectual content and approved the final version.

Funding Open Access funding enabled and organized by Projekt DEAL. The original study was supported by Lundbeck A/S, Denmark. The project was supported in part by grants from the Deutsche Forschungsgemeinschaft (DFG, German Research Foundation) - TRR 265 Project-ID 402170461 - Project ID-421888313, Project-ID 437718741 and GRK2350-1 Project-ID 324164820.

\section{Declarations}

Ethical statement All study procedures were in accordance with the Declaration of Helsinki. The study was approved by the ethics committee of the University of Heidelberg (Ethics Commission II: 2014-607N-MA).

Conflict of interest Outside the submitted work, Derik Hermann received honoraria for participating in advisory boards of the pharmaceutical companies Indivior, Camurus, and Servier. Falk Kiefer received honoraria as a consultant for Amomed, Desitin, Indivior, Lundbeck und Otsuka. We do not have further commercial or financial involvements that might present an appearance of a conflict of interest.

Open Access This article is licensed under a Creative Commons Attribution 4.0 International License, which permits use, sharing, adaptation, distribution and reproduction in any medium or format, as long as you give appropriate credit to the original author(s) and the source, provide a link to the Creative Commons licence, and indicate if changes were made. The images or other third party material in this article are included in the article's Creative Commons licence, unless indicated otherwise in a credit line to the material. If material is not included in the article's Creative Commons licence and your intended use is not permitted by statutory regulation or exceeds the permitted use, you will need to obtain permission directly from the copyright holder. To view a copy of this licence, visit http://creativecommons.org/licenses/by/4.0/.

\section{References}

Ambrogne JA (2002) Reduced-risk drinking as a treatment goal: what clinicians need to know. J Subst Abus Treat 22:45-53. https://doi. org/10.1016/s0740-5472(01)00210-0

Annis H, Graham JM, Davis CS (1987) Inventory of drinking situations (IDS): user's guide. Addiction Research Foundation, Toronto

Anton RF (2008) Naltrexone for the management of alcohol dependence. N Engl J Med 359:715-721. https://doi.org/10.1056/ NEJMct0801733

Babor TF, Higgins-Biddle JC, Saunders JB, Monteiro MG (2001) AUDIT. The alcohol use disorders identification test. Guidelines for Use in Primary Care, 2 edn. World Health Organization, Geneva, Switzerland

Bach P, Weil G, Pompili E, Hoffmann S, Hermann D, Vollstädt-Klein S, Mann K, Perez-Ramirez U, Moratal D, Canals S, Dursun SM, Greenshaw AJ, Kirsch P, Kiefer F, Sommer WH (2020) Incubation of neural alcohol cue reactivity after withdrawal and its blockade by naltrexone. Addict Biol 25:e12717. https://doi.org/10. $1111 / \mathrm{adb} .12717$ 
Bakeman R, Robinson BF (2005) Understanding statistics in the behavioral sciences. Psychology Press, New York. https://doi.org/10. 4324/9781410612625

Bart G, Schluger JH, Borg L, Ho A, Bidlack JM, Kreek MJ (2005) Nalmefene induced elevation in serum prolactin in normal human volunteers: partial kappa opioid agonist activity? Neuropsychopharmacology 30:2254-2262. https://doi.org/10. 1038/sj.npp.1300811

Batra A, Müller CA, Mann K, Heinz A (2016) Alcohol dependence and harmful use of alcohol. Dtsch Arztebl Int 113:301-310. https://doi. org/10.3238/arztebl.2016.0301

Beck AT, Ward CH, Mendelson M, Mock J, Erbaugh J (1961) An inventory for measuring depression. Arch Gen Psychiatry 4:561-571. https://doi.org/10.1001/archpsyc.1961.01710120031004

Bohn MJ, Krahn DD, Staehler BA (1995) Development and initial validation of a measure of drinking urges in abstinent alcoholics. Alcohol Clin Exp Res 19:600-606. https://doi.org/10.1111/j.15300277.1995.tb01554.x

Braus DF et al (2001) Alcohol-associated stimuli activate the ventral striatum in abstinent alcoholics. J Neural Transm 108:887-894. https://doi.org/10.1007/s007020170038

Castera P et al (2018) Nalmefene, given as needed, in the routine treatment of patients with alcohol dependence: an interventional, openlabel study in primary care. Eur Addict Res 24:293-303. https://doi. org/10.1159/000494692

Courtney KE, Schacht JP, Hutchison K, Roche DJO, Ray LA (2016) Neural substrates of cue reactivity: association with treatment outcomes and relapse. Addict Biol 21:3-22. https://doi.org/10.1111/ adb. 12314

de Laat B et al (2019) The kappa opioid receptor is associated with naltrexone-induced reduction of drinking and craving. Biol Psychiatry 86:864-871. https://doi.org/10.1016/j.biopsych.2019. 05.021

Drobes DJ, Thomas SE (1999) Assessing craving for alcohol. Alcohol Res Health 23:179-186

Online document. European Medicines Agency [EMA] (2013) EPAR summary for the public. Selincro. Nalmefen. EMA/826403/2012, EMEA/H/C/002583.

Everitt BJ, Robbins TW (2005) Neural systems of reinforcement for drug addiction: from actions to habits to compulsion. Nat Neurosci 8: 1481-1489. https://doi.org/10.1038/nn1579

Everitt BJ, Robbins TW (2016) Drug addiction: updating actions to habits to compulsions ten years on. Annu Rev Psychol 67:23-50. https:// doi.org/10.1146/annurev-psych-122414-033457

Gianoulakis C (2001) Influence of the endogenous opioid system on high alcohol consumption and genetic predisposition to alcoholism. J Psychiatry Neurosci 26:304-318

Grüsser SM et al (2004) Cue-induced activation of the striatum and medial prefrontal cortex is associated with subsequent relapse in abstinent alcoholics. Psychopharmacology 175:296-302. https://doi.org/ 10.1007/s00213-004-1828-4

Gual A, He Y, Torup L, van den Brink W, Mann K (2013) A randomised, double-blind, placebo-controlled, efficacy study of nalmefene, asneeded use, in patients with alcohol dependence. Eur Neuropsychopharmacol 23:1432-1442. https://doi.org/10.1016/j. euroneuro.2013.02.006

Heatherton TF, Kozlowski LT, Frecker RC, Fagerstrom KO (1991) The Fagerstrom test for nicotine dependence: a revision of the Fagerstrom Tolerance Questionnaire. Br J Addict 86:1119-1127. https://doi.org/10.1111/j.1360-0443.1991.tb01879.x

Heilig $M$ et al (2010) Translating the neuroscience of alcoholism into clinical treatments: from blocking the buzz to curing the blues. Neurosci Biobehav Rev 35:334-344. https://doi.org/10.1016/j. neubiorev.2009.11.018

Heinz A, Beck A, Grüsser SM, Grace AA, Wrase J (2009) Identifying the neural circuitry of alcohol craving and relapse vulnerability. Addict
Biol 14:108-118. https://doi.org/10.1111/j.1369-1600.2008.00136.

Heinz A et al (2020) Addiction research consortium: losing and regaining control over drug intake $(\mathrm{ReCoDe})$-from trajectories to mechanisms and interventions. Addict Biol 25:e12866. https://doi.org/10. 1111/adb. 12866

Hendershot CS, Wardell JD, Samokhvalov AV, Rehm J (2016) Effects of naltrexone on alcohol self-administration and craving: meta-analysis of human laboratory studies Addiction biology: Advanced online publication doi:https://doi.org/10.1111/adb.12425

Ingman $\mathrm{K}$ et al (2005) Prolonged central mu-opioid receptor occupancy after single and repeated nalmefene dosing. Neuropsychopharmacology 30:2245-2253. https://doi.org/10. 1038/sj.npp. 1300790

Jonas DE et al (2014) Pharmacotherapy for adults with alcohol use disorders in outpatient settings: a systematic review and meta-analysis. JAMA 311:1889-1900. https://doi.org/10.1001/jama.2014.3628

Lukas SE et al (2013) Extended-release naltrexone (XR-NTX) attenuates brain responses to alcohol cues in alcohol-dependent volunteers: a bold FMRI study. NeuroImage 78:176-185. https://doi.org/10. 1016/j.neuroimage.2013.03.055

MacKillop J (2006) Factor structure of the alcohol urge questionnaire under neutral conditions and during a cue-elicited urge state. Alcohol Clin Exp Res 30:1315-1321. https://doi.org/10.1111/j. 1530-0277.2006.00159.x

Mann K, Bladström A, Torup L, Gual A, van den Brink W (2013) Extending the treatment options in alcohol dependence: a randomized controlled study of as-needed nalmefene. Biol Psychiatry 73: 706-713. https://doi.org/10.1016/j.biopsych.2012.10.020

Mann K et al (2014) Predicting naltrexone response in alcohol-dependent patients: the contribution of functional magnetic resonance imaging. Alcohol Clin Exp Res 38:2754-2762. https://doi.org/10.1111/acer. 12546

Mann K, Torup L, Sørensen P, Gual A, Swift R, Walker B, van den Brink W (2016) Nalmefene for the management of alcohol dependence: review on its pharmacology, mechanism of action and meta-analysis on its clinical efficacy. Eur Neuropsychopharmacol 26:1941-1949. https://doi.org/10.1016/j.euroneuro.2016.10.008

Mellentin AI, Skøt L, Nielsen B, Schippers GM, Nielsen AS, Stenager E, Juhl C (2017) Cue exposure therapy for the treatment of alcohol use disorders: a meta-analytic review. Clin Psychol Rev 57:195-207. https://doi.org/10.1016/j.cpr.2017.07.006

Miyata $\mathrm{H}$ et al (2019) Nalmefene in alcohol-dependent patients with a high drinking risk: randomized controlled trial. Psychiatry Clin Neurosci 73:697-706. https://doi.org/10.1111/pen.12914

Mucha RF, Pauli P, Weber M, Winkler M (2008) Smoking stimuli from the terminal phase of cigarette consumption may not be cues for smoking in healthy smokers. Psychopharmacology 201:81-95. https://doi.org/10.1007/s00213-008-1249-x

Myrick H, Anton RF, Li X, Henderson S, Randall PK, Voronin K (2008) Effect of naltrexone and ondansetron on alcohol cue-induced activation of the ventral striatum in alcohol-dependent people. Arch Gen Psychiatry 65:466-475. https://doi.org/10.1001/archpsyc.65.4. 466

O'Malley SS, Jaffe AJ, Chang G, Schottenfeld RS, Meyer RE, Rounsaville B (1992) Naltrexone and coping skills therapy for alcohol dependence: a controlled study. Arch Gen Psychiatry 49:881887. https://doi.org/10.1001/archpsyc.1992.01820110045007

Peacock A et al (2018) Global statistics on alcohol, tobacco and illicit drug use: 2017 status report. Addiction 113:1905-1926. https://doi. org/10.1111/add.14234

Quelch DR et al (2017) Nalmefene reduces reward anticipation in alcohol dependence - an experimental fMRI study. Biol Psychiatry 81:941948. https://doi.org/10.1016/j.biopsych.2016.12.029

Raabe A, Grüsser SM, Wessa M, Podschus J, Flor H (2005) The assessment of craving: psychometric properties, factor structure and a 
revised version of the Alcohol Craving Questionnaire (ACQ). Addiction 100:227-234. https://doi.org/10.1111/j.1360-0443.2005. 00960.x

Rehm J, Shield KD, Rehm MX, Gmel G, Frick U (2012) Alcohol consumption, alcohol dependence and attributable burden of disease in Europe: potential gains from effective interventions for alcohol dependence. Centre for Addiction and Mental Health, Toronto, Canada

Rehm J, Allamani A., Elekes Z., Jakubczyk A., Manthey J., Probst C., Struzzo P., Della Vedova R., Gual A., Wojnar M. (2015) Alcohol dependence and treatment utilization in Europe - a representative cross-sectional study in primary care 16 doi:https://doi.org/10. 1186/s12875-015-0308-8

Reinert DF, Allen JP (2002) The Alcohol Use Disorders Identification Test (AUDIT): a review of recent research. Alcohol Clin Exp Res 26:272-279. https://doi.org/10.1111/j.1530-0277.2002.tb02534.x

Rösner S, Hackl-Herrwerth A, Leucht S, Vecchi S, Srisurapanont M, Soyka M (2010) Opioid antagonists for alcohol dependence. Cochrane Database Syst Rev. https://doi.org/10.1002/14651858. CD001867.pub3

Ross HE, Gavin DR, Skinner HA (1990) Diagnostic validity of the MAST and the alcohol dependence scale in the assessment of DSM-III alcohol disorders. J Stud Alcohol 51:506-513. https:// doi.org/10.15288/jsa.1990.51.506

Schacht JP, Anton RF, Myrick H (2013) Functional neuroimaging studies of alcohol cue reactivity: a quantitative meta-analysis and systematic review. Addict Biol 18:121-133. https://doi.org/10.1111/j.13691600.2012.00464.x

Schacht JP, Randall PK, Latham PK, Voronin KE, Book SW, Myrick H, Anton RF (2017) Predictors of naltrexone response in a randomized trial: reward-related brain activation, OPRM1 genotype, and smoking status Neuropsychopharmacol online publication doi: https://doi.org/10.1038/npp.2017.74

Scheurich A, Müller MJ, Anghelescu I, Lörch B, Dreher M, Hautzinger M, Szegedi A (2005) Reliability and validity of the form 90 interview. Eur Addict Res 11:50-56. https://doi.org/10.1159/000081417

Schultz W, Dayan P, Montague PR (1997) A neural substrate of prediction and reward. Science 275:1593-1599. https://doi.org/10.1126/ science. 275.5306 .1593

Sjoerds Z, de Wit S, van den Brink W, Robbins TW, Beekman ATF, Penninx BWJH, Veltman DJ (2013) Behavioral and neuroimaging evidence for overreliance on habit learning in alcohol-dependent patients. Transl Psychiatry 3:e337. https://doi.org/10.1038/tp.2013. 107

Skinner HA, Allen BA (1982) Alcohol dependence syndrome: measurement and validation. J Abnorm Psychol 91:199-209. https://doi.org/ 10.1037/0021-843x.91.3.199

Sobell MB, Sobell LC (1995) Controlled drinking after 25 years: how important was the great debate? Addiction 90:1149-1153. https:// doi.org/10.1080/09652149541392
Soyka M, Müller CA (2017) Pharmacotherapy of alcoholism — an update on approved and off-label medications. Expert Opin Pharmacother 18:1187-1199. https://doi.org/10.1080/14656566.2017.1349098

Spanagel R, Weiss F (1999) The dopamine hypothesis of reward: past and current status. Trends Neurosci 22:521-527. https://doi.org/10. 1016/S0166-2236(99)01447-2

Spanagel R, Zieglgansberger W (1997) Anti-craving compounds for ethanol: new pharmacological tools to study addictive processes. Trends Pharmacol Sci 18:54-59. https://doi.org/10.1016/S01656147(97)89800-8

Sullivan JT, Sykora K, Schneiderman J, Naranjo CA, Sellers EM (1989) Assessment of alcohol withdrawal: the revised clinical institute withdrawal assessment for alcohol scale (CIWA-Ar). Br J Addict 84:1353-1357. https://doi.org/10.1111/j.1360-0443.1989.tb00737. $\mathrm{x}$

Swift RM (2013) Naltrexone and nalmefene: any meaningful difference? Biol Psychiatry 73:700-701. https://doi.org/10.1016/j.biopsych. 2013.03.002

Tiffany ST, Conklin CA (2000) A cognitive processing model of alcohol craving and compulsive alcohol use. Addiction 95:145-153. https:// doi.org/10.1080/09652140050111717

van den Brink W, Aubin HJ, Bladstrom A, Torup L, Gual A, Mann K (2013) Efficacy of as-needed nalmefene in alcohol-dependent patients with at least a high drinking risk level: results from a subgroup analysis of two randomized controlled 6-month studies Alcohol. Alcohol 48:570-578. https://doi.org/10.1093/alcalc/agt061

Vollstädt-Klein S et al (2010) Initial, habitual and compulsive alcohol use is characterized by a shift of cue processing from ventral to dorsal striatum. Addiction 105:1741-1749. https://doi.org/10.1111/j.13600443.2010.03022.x

Vollstädt-Klein S et al (2019) The effects of nalmefene on emotion processing in alcohol use disorder - a randomized, controlled fMRI study. Eur Neuropsychopharmacol 29:1442-1452. https://doi.org/ 10.1016/j.euroneuro.2019.10.014

Volpicelli JR, Alterman AI, Hayashida M, O'Brien CP (1992) Naltrexone in the treatment of alcohol dependence. Arch Gen Psychiatry 49: 876-880. https://doi.org/10.1001/archpsyc.1992.01820110040006

Wiers CE, Heinz A (2015) Neurobiology of alcohol craving and relapse prediction. John Wiley \& Sons, West Sussex, Great Britain. doi: https://doi.org/10.1002/9781118472415.ch10

Wittchen H-U, Wunderlich U, Gruschwitz S, Zaudig M (1997) SKID-I. Strukturiertes Klinisches Interview für DSM-IV. Achse I: Psychische Störungen. Interviewheft und Beurteilungsheft. Hogrefe, Göttingen

Wrase J et al (2007) Dysfunction of reward processing correlates with alcohol craving in detoxified alcoholics. NeuroImage 35:787-794. https://doi.org/10.1016/j.neuroimage.2006.11.043

Publisher's note Springer Nature remains neutral with regard to jurisdictional claims in published maps and institutional affiliations. 